Pacific Journal of Mathematic 


\title{
PROJECTIVE AND INJECTIVE DISTRIBUTIVE LATTICES
}

\section{RAYMOND BALBES}

\begin{abstract}
This paper is concerned with the properties of projective and injective distributive lattices.

By considering the minimal Boolean extension of a distributive lattice $L$, the question of the injectivity of $L$ is transferred to the category of Boolean algebras, where a characterization is known. The result is that $L$ is injectivein the category of distributive lattices-if and only if it is a complete Boolean algebra.
\end{abstract}

The first section deals with a method of defining $E$-free sequences of elements in a distributive lattice. Roughly speaking, these are elements which satisfy a given set $E$ of inequalities and no others except consequences of $E$.

We prove that a finite distributive lattice is projective if and only if the sum of any two meet irreducible elements is meed irreducible. For the general case we show that a distributive lattice is projective if and only if it is generated by an $E$-free sequence, where $E$ is a certain set of one-sided inequalities.

The last section concerns the projectivity of Boolean algebras, chains, and direct products.

1. E-free distributive lattices. Throughout this paper $\left\{x_{i}\right\}, i \in I$, will denote a sequence of distinct variables.

Definition 1.1. An inequality in $\left\{x_{i}\right\}, i \in I$, is a formula of the form

$$
x_{i_{1}} \cdot \cdots \cdot x_{i_{n}} \leqq x_{j_{1}}+\cdots+x_{j_{m}} \cdot
$$

Definition 1.2. Suppose $\left\{a_{i}\right\}, i \in J$, is a sequence of elements of a distributive lattice and $I \subseteq J$. Then $\left\{a_{i}\right\}, i \in J$, satisfies the inequality (1) if $a_{i_{1}} \cdot \cdots \cdot a_{i_{n}} \leqq a_{j_{1}}+\cdots+a_{j_{m}}$. If $\dot{E}$ is a set of inequalities in $\left\{x_{i}\right\}, i \in I$, then $\left\{a_{i}\right\}, i \in J$, satisfies $E$ if it satisfies all members of $E$.

THEOREM 1.3. If $\left\{a_{i}\right\}, i \in J$, is a sequence of elements of a distributive lattice $L, I \subseteq J$, and $f: L \rightarrow M$ is a homomorphism into $a$ distributive lattice $M$, then if $\left\{a_{i}\right\}, i \in J$, satisfies a set $E$ of inequalities in $\left\{x_{i}\right\}, i \in I$, then $\left\{f\left(a_{i}\right)\right\}, i \in J$, satisfies $E$.

Proof. This follows from the fact that homomorphisms preserve order. 
DEFINITION 1.4. Let $E$ be a set of inequalities in $\left(x_{i}\right), i \in I$, and $e$ an inequality in $\left(x_{i}\right), i \in J$, where $I \subseteq J$. Then $e$ is said to be a consequence of $E$ if and only if whenever $\left\{a_{i}\right\}, i \in J$, is a sequence in a distributive lattice $L$ which satisfies $E$, then it satisfies $e$.

Definition 1.5. If $E$ is a set of inequalities in $\left\{x_{i}\right\}, i \in I$, then a sequence $\left\{a_{i}\right\}, i \in J, I \subseteq J$, is said to be $E$-free if and only if:

(i) $\left\{a_{i}\right\}, i \in J$, satisfies $E$.

(ii) If $\left\{a_{i}\right\}, i \in J$, satisfies an inequality $e$ in $\left(x_{i}\right), i \in I$, then $e$ is a consequence of $E$.

It is clear that if $\left\{a_{i}\right\}, i \in J$, is $E$-free and $e$ is a consequence of $E$, then $\left\{a_{i}\right\}, i \in J$, satisfies $e$.

TheOREM 1.6. Let $\left\{a_{i}\right\}, i \in I$ be a sequence in a distributive lattice. Then there exists a set $E$ of inequalities in $\left\{x_{i}\right\}, i \in I$, such that $\left\{a_{i}\right\}$, $i \in I$, is an E-free sequence.

Proof. Let

$$
E=\left\{x_{i_{1}} \cdots \cdot x_{i_{n}} \leqq x_{j_{1}}+\cdots+x_{j_{m}} \mid a_{i_{1}} \cdots \cdot a_{i_{n}} \leqq a_{j_{1}}+\cdots+a_{j_{m}}\right\} .
$$

Now $\left\{a_{i}\right\}, i \in I$, is $E$-free for it satisfies $E$ and if it satisfies an inequality $e$ in $\left\{x_{i}\right\}, i \in I$, then $e \in E$. It is trivial that $e \in E$, implies that $e$ is a consequence of $E$.

Lemma 1.7. A mapping $f$ of a set $G$ of generators of a distributive lattice $L$ into a distributive lattice $M$ can be extended to a homomorphism $f^{\prime}: L \rightarrow M$ if and only if for any finite nonempty subsets $S, T$ of $G$, whenever

$$
\pi(S) \leqq \Sigma(T)
$$

then

$$
\pi(f(S)) \leqq \Sigma(f(T)) \text { where } f(S)=\{f(s) \mid s \in S\}
$$

Proof. The necessity follows immediately. Now if $a \in L$, then $a=\sum_{i=1}^{n} \pi\left(S_{i}\right)$ where $S_{i}$ is a finite nonempty subset of $G$ for $1 \leqq i \leqq n$. Define $f^{\prime}: L \rightarrow M$ by $f(a)=\sum_{i=1}^{n} \pi\left(f\left(S_{i}\right)\right)$. Since $\sum_{i=1}^{n} \pi\left(S_{i}\right)=\sum_{j=1}^{m} \pi\left(T_{j}\right)$ is equivalent to a collection of relations of the form (2), which by hypothesis are preserved by $f$, the function $f^{\prime}$ is well defined. It is now easy to show that $f^{\prime}$ is a homomorphism and an extension of $f$.

THEOREM 1.8. If $\left\{a_{i}\right\}, i \in J$, generates a distributive lattice $L$ and $E$ is a set of inequalities in $\left\{x_{i}\right\}, i \in I, I \subseteq J$, then $\left\{a_{i}\right\}, i \in J$, is 
E-free if and only if

(i) $\left\{a_{i}\right\}, i \in J$, satisfies $E$.

(ii) whenever $\left\{b_{i}\right\}, i \in J$, is a sequence of elements of a distributive lattice $M$ such that $\left\{b_{i}\right\}, i \in J$, satisfies $E$, then there exists a homomorphism $f: L \rightarrow M$ such that $f\left(a_{i}\right)=b_{i}$.

Proof. For the necessity of (ii), let $M$ be such a distributive lattice. By Lemma 1.7, we need only show that

$$
\begin{aligned}
& a_{i_{1}} \cdots \cdot a_{i_{n}} \leqq a_{j_{1}}+\cdots+a_{j_{m}} \text { implies } \\
& b_{i_{1}} \cdots \cdot b_{i_{n}} \leqq b_{j_{1}}+\cdots+b_{j_{m}} .
\end{aligned}
$$

But if (4) holds then $\left\{a_{i}\right\}, i \in J$, satisfies

$$
e=x_{i_{1}} \cdot \cdots \cdot x_{i_{n}} \leqq x_{j_{1}}+\cdots+x_{j_{m}} .
$$

Since $\left\{a_{i}\right\}, i \in J$ is $E$-free, $e$ is a consequence of $E$. But $M$ satisfies $E$ so (5) holds. Conversely, suppose $\left\{a_{i}\right\}, i \in J$ satisfies an inequality $e$ in $\left\{x_{i}\right\}, i \in J$. To show $e$ is a consequence of $E$, let $\left\{b_{i}\right\}, i \in J$, be a sequence in a distributive lattice $M$ which satisfies $E$. By hypothesis there is a homomorphism $f: L \rightarrow M$ such that $f\left(a_{i}\right)=b_{i}$. Thus, by Theorem 1.3, $\left\{b_{i}\right\}, i \in J$, satisfies $E$. So $e$ is a consequence of $E$.

THEOREM 1.9. (Existence) If $E$ is a set of inequalities $\left\{x_{i}\right\}$, $i \in I$, and $J \supseteqq I$, then there exists an E-free sequence $\left\{A_{i}\right\}, i \in J$.

Proof. For each inequality $e=x_{i_{1}} \cdots x_{i_{n}} \leqq x_{j_{1}}+\cdots+x_{j_{m}}$ in $\left\{x_{i}\right\}, i \in I$, let

$$
\mathfrak{L}(e)=\left\{s \in 2^{J} \mid s\left(i_{1}\right)=\cdots=s\left(i_{n}\right)=1, s\left(j_{1}\right)=\cdots=s\left(j_{m}\right)=0\right\}
$$

and if $E$ is a set of such inequalities, let $\mathfrak{L}(E)=\bigcup_{e \in E} \mathfrak{L}(e)$. Let $A_{i}=\left\{s \in 2^{J} \mid s(i)=1, s \notin \mathbb{R}(E)\right\}$. Finally, for each $i \in J$, set $A_{i, 1}=A_{i}$ and $A_{i, 0}=2^{J}-A_{i}$.

We first show that

(i) If $\bigcap_{i} A_{i, s(i)}=\varnothing$ then $s \in \mathbb{R}(E)$.

(ii) Let $\left\{a_{i}\right\}, i \in J$, be a sequence of members of a ring of subsets of a set $X$ and let $e=x_{i_{1}} \cdots \cdots x_{i_{n}} \leqq x_{j_{1}}+\cdots+x_{j_{m}}$.

Then $\left\{a_{i}\right\}, i \in J$, satisfies $e$ if and only if $\bigcap_{i} a_{i, i(s)}=\varnothing$ for all $s \in \mathfrak{R}(e)$, where $a_{i, 1}=a_{i}$ and $a_{i, 0}=X-a_{i}$.

For (i) if $s \notin \mathbb{L}(E)$ then if $s(i)=1, s \in A_{i, 1}$ and if $s(i)=0, s \in A_{i, 0}$. Hence $s \in \bigcap_{i} A_{i, s(i)}$. For (ii) first suppose $\left\{a_{i}\right\}, i \in J$, satisfies $e$. Then $a_{i_{1}} \cap \cdots \cap a_{i_{n}} \cap a_{j_{1}}^{\prime} \cap \cdots \cap a_{j_{m}}^{\prime}=\varnothing$ and hence $\bigcap_{i} a_{i, s(i)}=\varnothing$ whenever $s \in \mathfrak{R}(e)$. Conversely, suppose there exists

$$
p \in a_{i_{1}} \cap \cdots \cap a_{i_{n}} \cap a_{j_{1}}^{\prime} \cap \cdots \cap a_{j_{m}}^{\prime} .
$$


Define $s \in 2^{J}$ by $s(i)=1$ if and only if $p \in a_{i}$. Then $s \in \mathbb{R}(e)$ but $p \in \bigcap_{i} a_{\left.i, s_{i}\right)}$.

Suppose

$$
x_{i_{1}} \cdot \cdots \cdot x_{i_{n}} \leqq x_{j_{1}}+\cdots+x_{j_{m}} \in E .
$$

Then $\bigcap_{k=1}^{n} A_{i_{k}} \subseteq \bigcup_{l=1}^{m} A_{j_{l}}$, for if $s \notin \mathbb{Q}(E)$ and $s\left(i_{1}\right)=\cdots=s\left(i_{n}\right)=1$ then $s\left(j_{l}\right)=1$ for some $l \in\{1, \cdots, m\}$. Hence $\left\{A_{i}\right\}, i \in J$, satisfies $E$. Now suppose $\left\{A_{i}\right\}, i \in J$, satisfies an inequality $e$ in $\left\{x_{i}\right\}, i \in J$ and $\left\{a_{i}\right\}, i \in J$, is a sequence in a distributive lattice $M$ which satisfies $E$. We can assume that $M$ is a ring of sets. Note that if we apply (ii) to $\left\{A_{i}\right\}, i \in J$, then (i) shows that every member $s$ of $\mathcal{L}(e)$ is in $\mathfrak{L}(E)$. Again by (ii), $\left\{a_{i}\right\}, i \in J$, will satisfy $e$ provided every member of $\mathcal{L}(e)$ is a member of $\mathfrak{L}\left(e^{\prime}\right)$ for some $e^{\prime} \in E$. But this follows since $\mathfrak{L}(e) \subseteq \mathbb{L}(E)$.

Definition 1.10. A distributive lattice is said to be E-free if it is generated by an $E$-free sequence.

By Theorem 1.6 every distributive lattice is $E$-free for some set $E$, and any $\varnothing$-free distributive lattice is free.

THEOREM 1.11. (Uniqueness) Let $E$ be a set of inequalities in $\left\{x_{i}\right\}, i \in I$. If $L$ and $M$ are distributive lattices generated by $E$-free sequences $\left\{a_{i}\right\}, i \in J$, and $\left\{b_{i}\right\}, i \in J$, where $I \cong J$, then $L \cong M$.

Proof. Follows immediately from Theorem 1.8.

The following type of theorem is easily proved: Suppose $L$ is generated by the $E$-free sequence $\left\{a_{i}\right\}, i \in I$, where the inequalities of $E$ are of the form $x_{i} \leqq x_{j}$. If $P$ and $Q$ are finite nonempty subsets of $\left\{a_{i}\right\}, i \in I$, and $\pi(P) \leqq \Sigma(Q)$, then there exist $a_{p} \in P$ and $a_{q} \in Q$ and a finite sequence $a_{p} \leqq a_{i_{1}} \leqq \cdots \leqq a_{i_{n}} \leqq a_{q}$ such that all of the inequalities $x_{p} \leqq x_{i_{1}}, \cdots, x_{i_{n}} \leqq x_{q}$ are in $E$. Also it can be shown that if $e$ is a consequence of $E$ then it is a consequence of a finite subset of $E$.

Again suppose $E$ is a set of inequalities in $\left\{x_{i}\right\}, i \in I$ and $\left\{A_{i}\right\}, i \in I$, is the $E$-free sequence as in the proof of Theorem 1.9. Let $L$ be the ring of sets generated by $\left\{A_{i}\right\}, i \in I$. Setting $X^{\prime}=\left\{s \in 2^{I} \mid s \notin \mathbb{R}(E)\right\}$, the following theorem can be proved by direct computation.

THEOREM 1.12. $F$ is a prime filter in $L$ if and only if $F$ is the filter generated by $\left\{A_{i} \mid s(i)=1\right\}$ for some $s \in X^{\prime}$.

Thus we obtain the following characterization of the Stone space of the $E$-free distributive lattice $L$. 
Theorem 1.13. The Stone space of $L$ is $X^{\prime}$ with $\left\{A_{i} \mid i \in I\right\}$, as a subbasis for its topology.

2. Definitions. The definitions in this section are of a universal nature, so we consider an arbitrary category of algebras.

Definition 2.1. An algebra $A$ is a retract of an algebra $A_{1}$ if there exist homomorphisms $f: A_{1} \rightarrow A$ and $g: A \rightarrow A_{1}$ such that $f g=I_{\Delta}$, the identity function on $A$.

Definition 2.2. An algebra $A$ is injective if for every pair of algebras $A_{1}$ and $A_{2}$, every homomorphism $h: A_{2} \rightarrow A$, and every monomorphism $g: A_{2} \rightarrow A_{1}$, there exists a homomorphism $f: A_{1} \rightarrow A$ such that $f g=h$.

Definition 2.3. An algebra $A$ is projective if for every pair of algebras $A_{1}$ and $A_{2}$, every homomorphism $h: A \rightarrow A_{2}$, and every epimorphism $f: A_{1} \rightarrow A_{2}$, there exists a homomorphism $g: A \rightarrow A_{1}$ such that $f g=h$.

The terms retract, injective, and projective, when prefixed by $(0,1)$-, will be taken in the category of distributive lattices with a smallest and a greatest element, and homomorphisms which preserve 0 and 1. Otherwise, the category will be distributive lattices. It is immediate that retracts of injective (projective) distributive lattices are injective (projective).

3. Injective distributive lattices. We make use of the following theorem, proved by Halmos [3; p. 141] in the category of Boolean algebras: A Boolean algebra is injective if and only if it is complete.

Lemma 3.1. A complete Boolean algebra $L$ is $(0,1)$-injective.

Proof. Let $M$ be a distributive lattice with 0 and 1 . Nerode has shown that there exists a Boolean algebra $A$, and a $(0,1)$-monomorphism $\varphi: M \rightarrow A$ such that $\varphi(M)$ Boolean generates $A . A$ is unique to within isomorphism, and is called the minimal Boolean extension of $M$ [4].

Now let $L_{1}$ and $L_{2}$ be distributive lattices with 0 and $1, h: L_{2} \rightarrow L$ a $(0,1)$-homomorphism, and $g: L_{2} \rightarrow L_{1}$ a $(0,1)$-monomorphism. Let $B, B_{1}$ and $B_{2}$ be the minimal Boolean extensions of $L, L_{1}, L_{2}$ and $\varphi, \varphi_{1}, \varphi_{2}$ the corresponding $(0,1)$-monomorphisms. By a theorem of Nerode [4, p. 399] there exists Boolean homomorphisms $h^{\prime}: B_{2} \rightarrow B$ and $g^{\prime}: B_{2} \rightarrow B_{1}$ such that $h^{\prime} \varphi_{2}=\varphi h$ and $g^{\prime} \varphi_{2}=\varphi_{1} g$. 


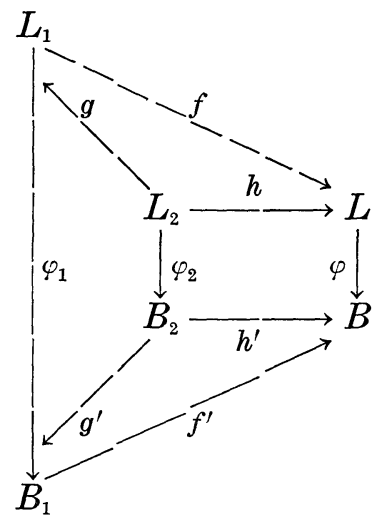

Furthermore since $g$ is one-to-one, so is $g^{\prime}$. By hypothesis, $L$ is a Boolean algebra, so $\varphi: L \rightarrow B$ is an isomorphism. Now since $B$ is complete, it is injective in the category of Boolean algebras. Therefore, there is a Boolean homomorphism $f^{\prime}: B_{1} \rightarrow B$ such that $f^{\prime} g^{\prime}=h^{\prime}$. Since $\varphi$ is an isomorphism, we can define $f: L_{1} \rightarrow L$ by $f=\varphi^{-1} f^{\prime} \varphi_{1}$. Then $f g=\varphi^{-1} f^{\prime} \varphi_{1} g=\varphi^{-1} f^{\prime} g^{\prime} \varphi_{2}=\varphi^{-1} h^{\prime} \varphi_{2}=\varphi^{-1} \varphi h=h$. Clearly $f$ preserves 0 and 1.

THEOREM 3.2. A distributive lattice is injective if and only if it is a complete Boolean algebra.

Proof. Suppose first that $L$ is a complete Boolean algebra. Let $L_{1}$ and $L_{2}$ be distributive lattices, $h: L_{2} \rightarrow L$ a homomorphism and $g: L_{2} \rightarrow L_{1}$ a monomorphism. Let $L_{1}^{\prime}=L_{1} \cup\left\{0^{\prime}, 1^{\prime}\right\}$ where $0^{\prime}<x<1^{\prime}$ for all $x \in L_{1}$, and $L_{2}^{\prime}=L_{2} \cup\left\{0^{*}, 1^{*}\right\}$ where $0^{*}<x<1^{*}$ for all $x \in L_{2}$. Define $h^{\prime}: L_{2}^{\prime} \rightarrow L$ by $h^{\prime} \mid L_{2}=h, h^{\prime}\left(0^{*}\right)=0_{L}$ and $h^{\prime}\left(1^{*}\right)=1_{L}$. Define $g^{\prime}: L_{2}^{\prime} \rightarrow L_{1}^{\prime}$ by $g^{\prime} \mid L_{2}=g, g^{\prime}\left(0^{*}\right)=0^{\prime}$ and $g^{\prime}\left(1^{*}\right)=1^{\prime}$. Since $L$ is a complete Boolean algebra, it is $(0,1)$-injective so there is a $(0,1)$ homomorphism $f^{\prime}: L_{1}^{\prime} \rightarrow L$ such that $f^{\prime} g^{\prime}=h^{\prime}$. Define $f: L_{1} \rightarrow L$ by $f=f^{\prime} \mid L_{1}$. Then if $x \in L_{2}, f g(x)=f^{\prime} g^{\prime}(x)=h^{\prime}(x)=h(x)$.

Conversely, suppose $L$ is injective and $B$ is the complete Boolean algebra of all subsets of the collection of prime filters of $L$. Then there exists a monomorphism $g: L \rightarrow B$. Since $L$ is injective there exists a homomorphism $f: B \rightarrow L$ such that $f g=I_{L}$. Thus $L$ is the homomorphic image of a Boolean algebra and is therefore a Boolean algebra. For completeness, let $S \subseteq L$. Then $\Sigma_{B}\{g(s) \mid s \in S\}=p$ exists in $B$. It is easily verified that $\Sigma_{L}(S)=f(p)$.

4. Basic properties of projective distributive lattices.

Lemma 4.1. A distributive lattice is projective if and only if it is a retract of a free distributive lattice. 
Proof. Essentially as in [3, p. 137].

Shanin $[5$, p. 91] has shown the topological dual of the statement that free Boolean algebras contain no uncountable chains. This implies the same condition of free distributive lattices, so we have:

THEOREM 4.2. There are no uncountable chains in a projective distributive lattice $L$.

Proof. Since $L$ is projective, there is a free distributive lattice $F$ and a monomorphism $g: L \rightarrow F$. If $C$ was an uncountable chain in $L,\{G(c) \mid c \in C\}$ would be an uncountable chain in $F$.

In the category of Boolean algebras, every projective Boolean algebra satisfies the $\omega$-chain condition. For distributive lattices there is an even stronger condition.

Definition 4.3. A subset $S$ of a distributive lattice $L$ is said to be $a$-disjointed $(a \in L)$ if $x y=a$ whenever $x$ and $y$ are distinct elements of $S$.

Lemma 4.4. Let $\left\{Z_{i}\right\}, i=1, \cdots, m$ and $\left\{T_{i}\right\}, i=1,2, \cdots \quad b e$ sequences of finite sets such that

$$
\begin{aligned}
& Z_{1} \nsubseteq T_{1}, \cdots, Z_{m} \nsubseteq T_{1} \\
& Z_{1} \nsubseteq T_{2}, \cdots, Z_{m} \nsubseteq T_{2} .
\end{aligned}
$$

Then there exists $i, j$ such that $i \neq j$, and $Z_{1} \nsubseteq T_{i} \cup T_{j}, \cdots, Z_{m} \nsubseteq T_{i} \cup T_{j}$.

Proof. The sequence $\left\{Z_{1}-T_{i}\right\}, i=1,2, \cdots$ contains only finitely many distinct sets since $Z_{1}$ is finite. So there exists a subsequence $\left\{T_{2, i}\right\}, i=1,2, \cdots$ of $\left\{T_{i}\right\}, i=1,2, \cdots$ such that $Z_{1}-T_{2,1}=Z_{1}$ $T_{2,2}=\cdots$. Hence $Z_{1} \nsubseteq T_{2,1} \cup T_{2,2} \cup \cdots$. Proceeding by induction, suppose $\left\{T_{n, i}\right\}, i=1,2, \cdots$ is a sequence such that $Z_{n}-T_{n, 1}=Z_{n}$ $T_{n, 2}=\cdots$. Now the sequence $\left\{Z_{n+1}-T_{n, i}\right\}, i=1,2, \cdots$ contains only finitely many distinct sets, so there is a subsequence $\left\{T_{n+1, i}\right\}, i=$ $1,2, \cdots$ of $\left\{T_{n, i}\right\}, i=1,2, \cdots$ such that $Z_{n+1}-T_{n+1,1}=Z_{n+1}-T_{n+1,2}=\cdots$. Hence $Z_{n+1} \nsubseteq T_{n+1,1} \cup T_{n+1,2} \cup \cdots$. In particular, $Z_{m} \nsubseteq T_{m, 1} \cup T_{m, 2} \cup \cdots$. Now for each $n \in\{1, \cdots, m\}, Z_{n} \nsubseteq T_{n, 1} \cup T_{n, 2} \cup \cdots$ and since $\left\{T_{m, i}\right\}, i=$ $1,2, \cdots$ is a subsequence of $\left\{T_{n, i}\right\}, i=1,2, \cdots$ we have $Z_{n} \nsubseteq T_{m, 1} \cup T_{m, 2}$ for all $n \in\{1, \cdots, m\}$.

LemmA 4.5. Let $F$ be a free distributive lattice generated by an independent set $G$. Then $\pi\left(S_{1}\right)+\cdots+\pi\left(S_{n}\right) \leqq \pi\left(T_{1}\right)+\cdots+\pi\left(T_{m}\right)$, where $S_{i}$ and $T_{j}$ are finite nonempty subsets of $G$ if and only if for each $i \in\{1, \cdots, n\}$ there exists $j \in\{1, \cdots, m\}$ such that $T_{j} \subseteq S_{i}$. 
Proof. The sufficiency follows immediately. On the other hand if there exists $p \in\{1, \cdots, n\}$ such that for each $j \in\{1, \cdots, m\}$ there is an element $t_{j} \in T_{j}-S_{p}$, then $\pi\left(S_{p}\right) \leqq t_{1}+\cdots+t_{m}$, contradicting independence.

THEOREM 4.6. In a free distributive lattice $L$, every a-disjointed subset is finite.

Proof. Let $L$ be generated by the independent set $G$ and suppose $D=\left\{d_{i} \mid i=1,2, \cdots\right\}$ is an infinite $a$-disjointed subset. There are finite nonempty subsets $S_{i, j}$ of $G$ such that $d_{i}=\sum_{j=1}^{p(i)} \pi\left(S_{i, j}\right)$. Let $a=\sum_{j=1}^{m} \pi\left(Z_{j}\right)$ where $Z_{j}$ is a finite nonempty subset of $G$ for $1 \leqq$ $j \leqq m$. We can assume $a \notin D$. Thus we have

(i ) $a<d_{i}(i=1,2, \cdots)$.

(ii) $d_{i} d_{j}=a$ whenever $i \neq j$.

(iii) There exists a positive integer $n$ such that for each $k \in\{1, \cdots, p(n)\}, Z_{r} \subseteq S_{n, k}$ for some $r \in\{1, \cdots, m\}$.

If (iii) does not hold then for each $i$ there is an $S_{i, j}$ such that $Z_{r} \nsubseteq S_{i, j}$ for all $r \in\{1, \cdots, m\}$. By Lemma 4.4 there exists $S_{i, j_{i}}$ and $S_{k, j_{k}}(i \neq k)$ such that $Z_{r} \nsubseteq S_{i, j_{i}} \cup S_{k, j_{k}}$ for all $r \in\{1, \cdots, m\}$. But $\pi\left(S_{i, j_{i}} \cup S_{k, j_{k}}\right) \leqq d_{i} d_{j}=a$, so by Lemma 4.5 there is an $r$ such that $Z_{r} \subseteq S_{i, j_{i}} \cup S_{k, j_{k}}$, a contradiction.

By (iii) we have $d_{n} \leqq a$, contradiction $(i)$.

CoRollary 4.7. In a projective distributive lattice every adisjointed set is finite.

Proof. Similar to the proof of Theorem 4.2.

EXAMPLE 4.8. Neither the ring of all sets of integers nor the ring of finite sets of integers is projective since the singletone form an infinite disjointed set.

EXAMPLE 4.9. The field of all finite and co-finite sets of integers is Boolean projective [3; p. 139, Corollary 2], but not projective.

5. Some characterizations of projective distributive lattices.

Theorem 5.1. A distributive lattice $L$ generated by an $E$-free sequence $\left\{a_{i}\right\}, i \in I$, is projective if and only if for each $i$ there exist finite nonempty subsets $S_{i, 1}, \cdots, S_{i, p(i)}$ of $I$ such that

(i) $a_{i}=\sum_{k=1}^{p(i)} P\left(S_{i, k}\right)$ where $P\left(S_{i, k}\right)=\pi\left\{a_{l} \mid l \in S_{i, k}\right\}$.

(ii) If

$$
x_{i_{1}} \cdot \cdots \cdot x_{i_{n}} \leqq x_{j_{1}}+\cdots+x_{j_{m}} \in E
$$

then for each $f \in \boldsymbol{P}\left(i_{1}, \cdots, i_{n}\right)$ there exist $q, r$ such that $S_{j_{q}, r} \subseteq$ 
$\mathbf{U}_{t=1}^{n} S_{i_{t}, f\left(i_{t}\right)}$ where $\boldsymbol{P}\left(i_{1}, \cdots, i_{n}\right)$ is the set of all function on $\left\{i_{1}, \cdots, i_{n}\right\}$ such that for each $t, f\left(i_{t}\right)$ is a positive integer $\leqq p\left(i_{t}\right)$.

Proof. Suppose $L$ is projective and $F$ is the free distributive lattice generated by the independent sequence $\left\{b_{i}\right\}, i \in I$. Let $h: F \rightarrow L$ be an epimorphism such that $h\left(b_{i}\right)=a_{i}$. By hypothesis there exists a homomorphism $g: L \rightarrow F$ such that $h g=I_{L}$. Let $g\left(a_{i}\right)=\sum_{k=1}^{p(i)} P_{F}\left(S_{i, k}\right)$ where for each $i$ and $k, S_{i, k}$ is a finite nonempty subset of $I$ and $P_{F}\left(S_{i, k}\right)=\left\{b_{l} \mid l \in S_{i, k}\right\}$. Then

$$
a_{i}=h g\left(a_{i}\right)=\sum_{k} h\left(P_{F}\left(S_{i, k}\right)\right)=\sum_{k} P\left(S_{i, k}\right) .
$$

Next suppose $x_{i_{1}} \cdot \cdots \cdot x_{i_{n}} \leqq x_{j_{1}}+\cdots+x_{j_{m}} \in E$. Since $\left\{a_{i}\right\}, i \in I$ is $E$-free, we have $a_{i_{1}} \cdots \cdot a_{i_{n}} \leqq a_{j_{1}}+\cdots+a_{j_{m}}$, so $g\left(a_{i_{1}}\right) \cdot \cdots \cdot g\left(a_{i_{n}}\right) \leqq$ $g\left(a_{j_{1}}\right)+\cdots+g\left(a_{j_{m}}\right)$. Thus for each $f \in \boldsymbol{P}\left(i_{1}, \cdots, i_{n}\right)$

$$
\begin{aligned}
P_{F}\left(\bigcup_{t=1}^{n} S_{i_{t}, f\left(i_{t}\right)}\right) & \leqq\left(\sum_{k} P_{F}\left(S_{i_{1}, k}\right)\right) \cdots\left(\sum_{k} P_{F}\left(S_{i_{n}, k}\right)\right) \\
& \leqq \sum_{k} P_{F}\left(S_{j_{1}, k}\right)+\cdots+\sum_{k} P_{F}\left(S_{j_{m}, k}\right) .
\end{aligned}
$$

By Lemma 4.5, we have that for each $f$, there exist $q, r$ such that $S_{j_{q}, r} \subseteq \bigcup_{t=1}^{n} S_{i_{t}, f\left(i_{t}\right)}$.

Now suppose (i) and (ii) hold and again let $F$ be the free distributive lattice generated by the independent sequence $\left\{b_{i}\right\}, i \in I$, and $h: F \rightarrow L$ an epimorphism such that $h\left(b_{i}\right)=a_{i}$. Define a sequence $\left\{c_{i}\right\}, i \in I$ in $F$ by $c_{i}=\sum_{k=1}^{p(i)} P_{F}\left(S_{i, k}\right)$ for each $i \in I$. We will show that $\left\{c_{i}\right\}, i \in I$ satisfies $E$. If $x_{i_{1}} \cdots \cdot x_{i_{n}} \leqq x_{j_{1}}+\cdots+x_{j_{m}} \in E$, then by hypothesis, for each $f \in \boldsymbol{P}\left(i_{1}, \cdots, i_{n}\right)$, there exist $q, r$ such that $S_{j_{q}, r} \leqq \bigcup_{t=1}^{n} S_{i_{t}, f\left(i_{t}\right)}$. So $P_{F}\left(\bigcup_{t=1}^{n} S_{i_{t} f\left(i_{t}\right)}\right) \leqq P_{F}\left(S_{j_{q}, r}\right) \leqq c_{j_{q}} \leqq c_{j_{1}}+\cdots+c_{j_{m}}$. Thus,

$$
\begin{aligned}
c_{i_{1}} \cdots \cdot c_{i_{n}} & =\left(\sum_{k} P_{F}\left(S_{i_{1}, k}\right)\right) \cdot \cdots \cdot\left(\sum_{k} P_{F}\left(S_{i_{n}, k}\right)\right) \\
& =\sum_{f} P_{F}\left(\bigcup_{t=1}^{n} S_{i_{t}, f\left(i_{t}\right)}\right) \leqq c_{j_{1}}+\cdots+c_{j_{m}} .
\end{aligned}
$$

Since $\left\{c_{i}\right\}, i \in I$ satisifies $E$, by Theorem 1.8, there exists a homomorphism $g: L \rightarrow F$ such that $g\left(a_{i}\right)=c_{i}$. Hence $h g\left(a_{i}\right)=h\left(c_{i}\right)=\sum_{k=1}^{p(i)} P\left(S_{i, k}\right)=a_{i}$. By Lemma 4.1, $L$ is projective.

It may be remarked that for each $i$, the sets $S_{i, 1}, \cdots, S_{i, p(i)}$ may be chosen so that no one of them contains any other one.

CoRollary 5.2. Suppose $L$ is a distributive lattice generated by a sequence $\left\{a_{i}\right\}, i \in I$. Then $L$ is projective if and only if for each $i$, there exists finite nonempty subsets $S_{i, 1}, \cdots, S_{i, p(i)}$ of the distinct 
elements of $\left\{a_{i}\right\}, i \in I$, such that

(i) $\quad a_{i}=\sum_{k=1}^{p(i)} \pi\left(S_{i, k}\right)$.

(ii) If $a_{i_{1}} \cdots a_{i_{n}} \leqq a_{j_{1}}+\cdots+a_{j_{m}}$, then for each $f \in \boldsymbol{P}\left(i_{1}, \cdots, i_{n}\right)$, there exist $q, r^{*}$ such that $S_{\jmath_{q}, r} \subseteq \bigcup_{t_{1}^{n}}^{n} S_{i_{t}, f\left(i_{t}\right)}$, where $\boldsymbol{P}\left(i_{1}, \cdots, i_{n}\right)$ is the set of all functions on $\left\{i_{1}, \cdots, i_{n}\right\}$ such that for each $t, f\left(i_{t}\right)$ is a positive integer $\leqq p\left(i_{t}\right)$.

Proof. This follows immediately from Theorem 5.1 by defining $E$ as in Theorem 1.6 .

In Theorem 5.1 and Corollary 5.2, we shall refer to condition (i) as the projective representation of the element $a_{i}$, and to (ii) as the projective criterion for $x_{i_{1}} \cdots \cdot x_{i_{n}} \leqq x_{j_{1}}+\cdots+x_{j_{m}}\left(a_{i_{1}} \cdots \cdot a_{i_{n}} \leqq\right.$ $\left.a_{j_{1}}+\cdots+a_{j_{m}}\right)$. Observe that in (ii) of Corollary 5.2, if $a_{i_{p}}=a_{j_{q}}$ for some $i_{p}$ and $j_{q}$, then the criterion is automatically satisfied. From this we again see that free distributive lattices are projective.

DeFinition 5.3. An inequality $e=x_{i_{1}} \cdots x_{i_{n}} \leqq x_{j_{1}}+\cdots+x_{j_{m}}$ in $\left\{x_{i}\right\}, i \in I$, is said to be one-sided if $n=1$ or $m=1$. If $G=\left\{a_{i} \mid i \in I\right\}$, is a subset of a distributive lattice then $G$ is said to be lower semiindepenaient (upper semi-idependent) if whenever $G$ satisfies $e$ then there exists $p \in\{1, \cdots, n\}$ such that $a_{i_{p}} \leqq a_{j_{1}}+\cdots+a_{j_{m}}$ (there exists $q \in\{1, \cdots, m\}$ such that $a_{i_{1}} \cdots \cdot a_{i_{n}} \leqq a_{j_{q}}$ ).

For the following theorem $\left\{x_{i}\right\}, i \in I$, will be, as before, a sequence of distinct variables. Fix a definite simple ordering of $I$. If $i_{1}<\cdots<i_{n}$ and $X=\left\{x_{i_{1}}, \cdots, x_{i_{n}}\right\}$, then $\pi(X)$ will denote the expression $x_{i_{1}} \cdots \cdots x_{i_{n}}$ and $\Sigma(X)$ will denote $x_{i_{1}}+\cdots+x_{i_{n}}$ 。

THEOREM 5.4. Let $L$ be a distributive lattice generated by $\left\{a_{2}\right\}$, $i \in I$. If $L$ is projective then $\left\{a_{i}\right\}, i \in I$, is E-free for some set $E$ of one-sided inequalities. Specifically, $L$ is projective if and only if $\left\{a_{i}\right\}, i \in I$, is E-free for some set $E$ of inequalities of the form $E_{1} \cup E_{2}$ where

$$
\begin{aligned}
& E_{1}=\bigcup_{i \in I}\left\{\pi\left(X_{i, j}\right) \leqq x_{i} \mid j=1, \cdots, p(i)\right\} \text { and } \\
& E_{2}=\bigcup_{i \in I}\left\{x_{\imath} \leqq \Sigma\left(Y_{i, j}\right) \mid j=1, \cdots, q(i)\right\} \text { and }
\end{aligned}
$$

(i) $X_{i, j}$ is a finite subset of $\left\{x_{i} \mid i \in I\right\}, 1 \leqq j \leqq p(i)$.

(ii) For each $i$ : $Y_{i, 1}, \cdots, Y_{i, q(i)}$ are all possible sets of the form $\left\{x_{i_{1}}, \cdots, x_{i_{n}}\right\}$ where $x_{i_{j}} \in X_{i, j}$.

(iii) If $x_{i_{1}} \cdots \cdots x_{n_{n}} \leqq x_{j_{1}}+\cdots+x_{j_{m}} \in E$ (so that $n=1$ or $m=1$ ), then for each $f \in \boldsymbol{P}\left(i_{1}, \cdots, i_{n}\right)$, there exist $q, r$ such that $X_{j_{q}, r} \leqq$ $\bigcup_{t=1}^{n} X_{i_{t}, f\left(i_{t}\right)}$. 
Proof. For sufficiency, let

$$
S_{i, j}=\left\{k \mid x_{k} \in X_{i, j}\right\} \text {, and } T_{i, j}=\left\{k \mid x_{k} \in Y_{i, j}\right\} \text {. }
$$

Since $\left\{a_{i}\right\}, i \in I$, satisfies $E$, for each $i$, we have $\pi\left\{a_{k} \mid k \in S_{i, j}\right\} \leqq a_{i}$ for $j=1, \cdots, p(i)$. Therefore $\sum_{j=1}^{p(i)} \pi\left\{a_{k} \mid k \in S_{i, j}\right\} \leqq a_{i}$. Similarly, $a_{i} \leqq$ $\sum\left\{a_{k} \mid k \in T_{i, j}\right\}$ for $j=1, \cdots, q(i)$. A simple calculation shows that $a_{i}=$ $\sum_{j=1}^{p(i)} P\left(S_{i, j}\right)$ where $P\left(S_{i, j}\right)=\pi\left\{a_{k} \mid k \in S_{i, j}\right\}$. Since $X_{j_{q}, r} \subseteq \bigcup_{t=1}^{n} X_{i_{t}, f\left(i_{t}\right)}$ implies $S_{j_{q}, r} \subseteq \bigcup_{t=1}^{n} S_{i_{t}, f\left(i_{t}\right)}, L$ is projective by Theorem 5.1.

For the necessity we use Theorem 5.2. Thus, let $a_{i}=\sum_{k=1}^{p(i)} \pi\left(S_{i, j}\right)$ be a projective representation for each $i$. Then for each $i$ we have $\pi\left(S_{i, j}\right) \leqq a_{i}$ for $j=1, \cdots, p(i)$ and $a_{i} \leqq \Sigma\left(T_{i, j}\right)$ for $j=1, \cdots, q(i)$ where $T_{i, 1}, \cdots, T_{i, q(i)}$ are all possible sets of the form $\left\{a_{i_{1}}, \cdots, a_{i_{n}}\right\}$ and $a_{i_{j}} \in S_{i, j}$. Setting $X_{i, j}=\left\{x_{k} \mid a_{k} \in S_{i, j}\right\}$ and $Y_{i, j}=\left\{x_{k} \mid a_{k} \in T_{i, j}\right\}$, define $E$ as in the statement of the theorem. Consequently (i) and (ii) are satisfied. For (iii) suppose $x_{i_{1}} \cdots \cdot x_{i_{n}} \leqq x_{j_{1}}+\cdots+x_{j_{m}} \in E$. By the definitions of $S_{i, j}, T_{i, j}, X_{i, j}$, and $Y_{i, j}$, we have $a_{i_{1}} \cdots a_{i_{n}} \leqq a_{j_{1}}+\cdots+a_{j_{m}}$. But $L$ is projective so for each $f \in \boldsymbol{P}\left(i_{1}, \cdots, i_{n}\right)$ there exist $q, r$ such that $S_{j_{q}, r} \leqq \bigcup_{t=1}^{n} S_{i_{t}, f\left(i_{t}\right)}$. Hence $X_{j_{q}, r} \subseteq \mathbf{U}_{t=1}^{n} X_{i_{t}, f\left(i_{t}\right)}$.

It remains to show that $\left\{a_{i}\right\}, i \in I$, is $E$-free. First, $\left\{a_{i}\right\}, i \in I$, obviously satisfies $E$. Now suppose $\left\{a_{i}\right\}, i \in I$, satisfies an inequality $e=x_{i_{1}} \cdots \cdot x_{i_{n}} \leqq x_{j_{1}}+\cdots+x_{j_{m}}$. Then $a_{i_{1}} \cdots \cdot a_{i_{n}} \leqq a_{j_{1}}+\cdots+a_{j_{m}}$. Since $L$ is projective, for each $f \in \boldsymbol{P}\left(i_{1}, \cdots, i_{n}\right)$ there exist $q, r$ such that $S_{j_{q}, r} \subseteq \mathbf{U}_{t=1}^{n} S_{i_{t}, f\left(i_{t}\right)}$. Hence

$$
X_{j_{q}, r} \subseteq \bigcup_{t=1}^{n} X_{i_{t}, f\left(i_{t}\right)}
$$

To show $e$ is a consequence of $E$, let $\left\{b_{i}\right\}, i \in I$, be a sequence in a distributive lattice that satisfies $E$. Let $B_{i, j}=\left\{b_{k} \mid x_{k} \in X_{i, j}\right\}$. Then $b_{i}=\sum_{k=1}^{p(i)} \pi\left(B_{i, k}\right)$ and by $(6)$, for each $f \in \boldsymbol{P}\left(i_{1}, \cdots, i_{n}\right)$ there exist $q, r$ such that $B_{j_{q}, r} \subseteq \bigcup_{t=1}^{n} B_{i_{t}, f\left(i_{t}\right)}$. Hence $\pi\left(\bigcup_{t=1}^{n} B_{i_{t}, f\left(i_{t}\right)}\right) \leqq \pi\left(B_{j_{q}, r}\right) \leqq b_{j_{q}}$. Thus

$$
\begin{aligned}
b_{i_{1}} \cdot \cdots \cdot b_{i_{n}} & =\left(\sum_{k} \pi\left(B_{i_{1}, k}\right)\right) \cdot \cdots \cdot\left(\sum_{k} \pi\left(B_{i_{n}, k}\right)\right) \\
& \left.=\sum_{f} \pi\left(\bigcup_{f}^{n} B_{i_{t}, f\left(i_{t}\right)}\right)\right) \leqq b_{j_{1}}+\cdots+b_{j_{m}} .
\end{aligned}
$$

So $e$ is a consequence of $E$, and $\left\{a_{i}\right\}, i \in I$, is $E$-free.

EXAMPLE 5.5. Let $\left\{x_{i}\right\}, i=1,2, \cdots$ be a sequence of distinct variables and $E=\left\{x_{1} x_{2} \leqq x_{3}+x_{4}\right\}$. Then any $E$-free distributive lattice is nonprojective for $x_{1} x_{2} \leqq x_{3}+x_{4}$ is not a consequence of one-sided inequalities.

EXAMPLE 5.6. Let $\left\{x_{i}\right\}, i=1,2, \cdots$ be a sequence of distinct 
variables and

$$
E=\left\{x_{1} x_{2} \leqq x_{3}, x_{1} x_{2} \leqq x_{4}, x_{3} \leqq x_{4}+x_{5}, x_{1} \leqq x_{4}+x_{5}\right\} .
$$

Then the distributive lattice generated by the $E$-free sequence $\left\{a_{i}\right\}$, $i \in I$, is projective for a projective representation is: $a_{1}=a_{1} a_{4}+a_{1} a_{5}$, $a_{2}=a_{2}, a_{3}=a_{3} a_{4}+a_{3} a_{5}+a_{1} a_{2}, a_{4}=a_{4}+a_{1} a_{2}$, and $a_{i}=a_{i}$ for $i \geqq 5$.

\section{Meet and join irreducible elements.}

Definition 6.1. An element $a$ of the lattice $L$ is called meet irreducible (M.I.) if whenever $x y \leqq a$ then $x \leqq a$ or $y \leqq a$. Join irreducible (J.I.) elements are defined dually.

In a distributive lattice the following are equivalent

(i) $a$ is M.I.

(ii) If $a_{1} \cdots \cdots a_{n} \leqq a$ then $a_{i} \leqq a$ for some $i \in\{1, \cdots, n\}$.

(iii) If $a_{1} \cdots \cdots a_{n}=a$ then $a_{i}=a$ for some $i \in\{1, \cdots, n\}$.

THEOREM 6.2. In a projective distributive lattice the sum of any two meet irreducible elements is meet irreducible and the product of any two join irreducible elements is join irreducible.

Proof. Let $L=\left\{a_{i},\right\} i \in I$ and suppose $a_{1}$ and $a_{2}$ are M.I. and

$$
a_{3} \alpha_{4} \leqq a_{1}+a_{2} \text {. }
$$

Let $a_{i}=\sum_{j=1}^{p(i)} \pi\left(S_{i, j}\right)$ be a projective representation for each $i$. If $a_{3} \not \equiv a_{1}+a_{2}$ and $a_{4} \not \equiv a_{1}+a_{2}$ then there exist integers $m, n$ such that $\pi\left(S_{3, m}\right) \nRightarrow a_{1}+a_{2}$ and $\pi\left(S_{4, m}\right) \geqq a_{1}+a_{2}$. For otherwise, for either $k=1$ or $k=2, \pi\left(S_{k, t}\right) \leqq a_{1}+a_{2}$ for all $t \in\{1, \cdots, p(k)\}$ and so $a_{k} \leqq a_{1}+a_{2}$. Now choosing $f \in \boldsymbol{P}(3,4)$ such that $f(3)=m$ and $f(4)=n$, we have

$$
\pi\left(S_{t, f(t)}\right) \not a_{1}+a_{2} \text { for } t=3,4 .
$$

By the criterion (applied to (7) and for the given $f$ ), there exist $q \in\{1,2\}$ and $r \in\{1, \cdots, p(q)\}$ such that $S_{q, r} \subseteq S_{3, f(3)} \cup S_{4, f(4)}$. Hence $\pi\left(S_{3, f(3)}\right) \pi\left(S_{4, f(4)}\right) \leqq \pi\left(S_{q, r}\right) \leqq a_{q}$. But $a_{q}$ is M.I. so for either $n=3$ or $n=4, \pi\left(S_{n, f(n)}\right) \leqq a_{q} \leqq a_{1}+a_{2}$, contradicting (8).

Since the dual of a projective distributive lattice is projective, the second statement follows.

7. Finite projective distributive lattices. We will now choose a special set of generators and apply Theorem 5.2. In particular, recall that in a finite distributive lattice every element is a product of M.I. elements. 
THEOREM 7.1. In a finite distributive lattice $L$, the following are equivalent.

(i ) $L$ is projective.

(ii ) $L$ is generated by a lower semi-independent set.

(ii') $L$ is generated by an upper semi-independent set.

(iii) The sum of any two meet irreducible elements is meet irreducible.

(iii') The product of any two join irreducible elements is join irreducible.

Proof. (i) $\Rightarrow$ (iii) and (i) $\Rightarrow$ (iii') follows from Theorem 6.2. (iii) $\Rightarrow$ (ii): Let $G$ be the set of M.I. elements. Then $G$ generates $L$. If

$$
a_{i_{1}} \cdot \cdots \cdot a_{i_{n}} \leqq a_{j_{1}}+\cdots+a_{j_{m}}
$$

where $a_{i_{s}}, a_{j_{t}} \in G$ then by (iii) $a_{j_{1}}+\cdots+a_{j_{m}}$ is M.I., so there exists $p \in\{1, \cdots, n\}$ such that $a_{i p} \leqq a_{j_{1}}+\cdots+a_{j_{m}}$. (iii') $\Rightarrow$ (ii'): This is the dual of (iii) $\Rightarrow$ (ii). (ii') $\Rightarrow$ (i). For each $a_{i} \in G$, it will be proved that a projective repesentation is $a_{i}=\pi\left(S_{i, 1}\right)+\cdots+\pi\left(S_{i, p(i)}\right)$ where the $S_{i, j}$ are all possible sets such that $\pi\left(S_{i, j}\right) \leqq a_{i}$. Equality holds since one of these sets is $\left\{a_{i}\right\}$. To show the criterion is satisfied, suppose $a_{i_{1}} \cdots \cdot a_{i_{n}} \leqq a_{j_{1}} \cdots \cdot a_{j_{m}}$. Then there exists $q$ such that $a_{i_{1}} \cdot \cdots \cdot a_{i_{n}} \leqq a_{j_{q}}$. Let $f \in \boldsymbol{P}\left(i_{1}, \cdots, i_{n}\right)$, then

$$
\pi\left(\bigcup_{t=1}^{n} S_{i_{t}, f\left(i_{t}\right)}\right)=\prod_{t=1}^{n} \pi\left(S_{i_{t}, f\left(i_{t}\right)}\right) \leqq a_{i_{1}} \cdots \cdot a_{i_{n}} \leqq a_{j_{q}} \cdot
$$

But by the definition of $S_{j_{q}, 1}, S_{j_{q}, 2}, \cdots$ there is an $r$ such that $S_{j_{q}, r}=$ $\mathrm{U}_{t=1}^{n} S_{i_{t}, f\left(i_{t}\right)}$. (ii) $\Rightarrow$ (i): Since (ii') $\Rightarrow$ (i), by duality if (ii) then the dual of $L$ is projective and hence $L$ is projective.

The hypothesis of finiteness is essential for the J.I. elements in ring of subsets of the integers are the singletons and $\varnothing$. So (iii') is satisfied but we have seen (Ex. 4.8) that this lattice is not projective.

EXAMPLE 7.2. Let $f$ be the free distributive lattice generated by the independent set $\left\{a_{1}, a_{2}, a_{3}\right\}$. Then the sublattice

$$
L_{1}=\left\{a_{1} a_{2} a_{3}, a_{1} a_{2}, a_{1} a_{3}, a_{1}\left(a_{2}+a_{3}\right), a_{1}, a_{2}+a_{3}, a_{1}+a_{2}+a_{3}\right\}
$$

is not projective for $a_{1}$ and $a_{2}+a_{3}$ are J.I. in $L_{1}$ but $a_{1} a_{2}+a_{1} a_{3}$ is not J.I. in $L_{1}$.

By considering the partially ordered set of nonzero J.I. elements of a finite distributive lattice we obtain the following theorem [1, p. 139].

THEOREM 7.3. Every finite distributive lattice is isomorphic with the family of all hereditary subsets of partially ordered set. 
Conversely, the family of all hereditary subsets of a finite partially ordered set is a lattice.

By adding a 0 and 1 to the partially ordered set, we find that every finite distributive lattice is isomorphic with the family of all nonempty proper hereditary subsets of a partially ordered set with 0,1 . Conversely, every such family is a distributive lattice. In contrast, for finite projective distributive lattices we have:

THEOREM 7.4. Every finite projective distributive lattice is isomorphic with the family of all nonempty proper hereditary subsets of a finite lattice. Conversely, the family of all nonempty proper hereditary subsets of a finite lattice is a projective distributive lattice.

Proof. Let $L$ be a finite projective distributive lattice and $P$ the set of all nonzero J.I. elements. Then $L$ is isomorphic with the family of hereditary subsets of $P$. Let $M=\{0\} \cup P \cup\left\{1^{*}\right\}$ where $1^{*}>x$ for all $x \in L$. Now $M$ will be a lattice if $S \subseteq M$ implies $\pi(S)$ exists. If $S=\varnothing$ then $\pi(S)=1^{*} \in M$ and if $S \neq \varnothing$ it is sufficient to consider $S=\{x, y\}$. If $x$ or $y$ equals 0 then $\pi(S)=0$ and if $x$ or $y$ equals $1^{*}$ then $\pi(S)$ equals $y$ or $x$ respectively. Thus, suppose $x, y \in P$. Since $x, y$ are J.I. and $L$ is projective $x \cdot{ }_{L} y \in P \cup\{0\}$ and therefore is equal to $x \cdot{ }_{M} y$. Hence, $M$ is a lattice. Finally, the lattice of hereditary subsets of $P$ is isomorphic with the lattice of nonempty proper hereditary subsets of $M$ under the correspondence $H \in P \leftrightarrow\{0\} \cup H \in M$.

Now suppose $L$ is the family of all nonempty proper hereditary subsets of a finite lattice $M$. Clearly $L$ is a distributive lattice. Let $L_{1}$ be the set of all hereditary subsets of $M$; then $L_{1}=\{\varnothing, M\} \cup L$. By the proof of [1, Th. 5, p. 139], the set of all nonzero J.I. elements of $L_{1}$ is isomorphic with the collection of principal ideals of $M$. Therefore, the set of J.I. elements of $L$ is isomorphic with the set consisting of $\varnothing$ and all proper principal ideals of $M$, and is therefore closed under products. Hence $L$ is projective.

\section{Applications and Examples.}

THEOREM 8.1. Boolean algebras and Boolean rings are projective if and only if they are finite.

Proof. Infinite Boolean algebras and rings contain infinite disjointed sets, and hence can not be projective. On the other hand, a finite Boolean ring is a Boolean algebra and every finite Boolean algebra is isomorphic with the collection of all subsets of a finite 
set. Clearly the J.I. elements-the singletons and $\varnothing$-are closed under products.

THEOREm 8.2. A chain is projective if and only if it is countable.

Proof. Theorem 4.2 shows the necessity. Now suppose $C=$ $\left\{a_{i} \mid i=1,2, \cdots\right\}$ is a chain. It will be shown that $C$ is a retract of the free distributive lattice $F$ generated by the independent set $\left\{b_{i} \mid i=\right.$ $1,2, \cdots\}$. Let $f: F \rightarrow C$ be an epimorphism such that $f\left(b_{i}\right)=a_{i}$ for $i=1,2, \cdots$. Define,inductively, a function $g: C \rightarrow F$ by $g\left(a_{1}\right)=b_{1}$ and

$$
g\left(a_{n}\right)=b_{n} \pi\left\{g\left(a_{i}\right) \mid a_{i}>a_{n}, i<n\right\}+\Sigma\left\{g\left(a_{i}\right) \mid a_{i}<a_{n}, i<n\right\} .
$$

Then $g$ is a homomorphism and $f g=I_{\sigma}$.

ExAMPLE 8.3. Let $C$ be the chain of nonnegative integers. Then $C \times C$ is not projective.

Proof. $C \times C$ is generated by the elements $a_{i}=(i, 0)$ and $b_{j}=$ $(0, j)$ where $i>0, j>0$. If $C \times C$ is projective then there exists a projective representation:

$$
\begin{aligned}
& a_{i}=\pi\left(S_{i, 1}\right)+\cdots+\pi\left(S_{i, p(i)}\right)(i>0) \\
& b_{j}=\pi\left(T_{j, 1}\right)+\cdots+\pi\left(T_{j, p(j)}\right)(j>0) .
\end{aligned}
$$

Now for some $r$, say $r=1, S_{i, r}$ is of the form $S_{i, r}=\left\{a_{i_{1}}, \cdots, a_{i_{n}}\right\}$ where $i \leqq i_{1}<\cdots<i_{n}$. For if not, by distributivity, we have $a_{i} \leqq$ $c_{1}+\cdots+c_{p(i)}$, where for each $r, c_{r} \in S_{i, r}$ and either $c_{r}=b_{j}$ for some $j$ or $c_{r}=a_{k}$ for some $k<i$. This is impossible, as is seen by comparing first coordinates. Similarly, we may assume $T_{j, 1}=\left\{b_{j_{1}}, \cdots, b_{j_{m}}\right\}$, where $j \leqq j_{1}<\cdots<j_{m}$.

Now let $p$ be an integer larger than the subscripts of all elements $a_{i}$ or $b_{j}$ which occur in the projective representation of $a_{1}$. Since $a_{p} b_{p} \leqq a_{1}$, by the projective criterion, $S_{p, 1} \cup T_{p, 1} \supseteqq S_{1, i}$ for some $i$. This is a contradiction.

THEOREM 8.4. The direct product $\prod_{i \in I} L_{i}$ of finite distributive lattices is projective if and only if $L_{i}$ is projective for each $i \in I$ and $\left|L_{i}\right|=1$ for all but finitely many $i \in I$.

Proof. Suppose the condition holds. Then it is sufficient to show that if $L_{1}$ and $L_{2}$ are projective then $L_{1} \times L_{2}$ is projective. It is easily verified that the M.I. elements of $L_{1} \times L_{2}$ are those of the form $(x, y),(x, 1)$ and $(1, y)$ where $x$ and $y$ are M.I. in $L_{1}$ and $L_{2}$ respectively. But since the M.I. elements of $L_{1}\left(L_{2}\right)$ are closed under sums, the M.I. elements of $L_{1} \times L_{2}$ also have this property. Hence $L_{1} \times L_{2}$ is projective. Conversely, each $L_{i}$ is a retract of $\Pi_{i \in I} L_{i}$ and 
is therefore projective. If $L_{i}>1$ for infinitely many $i \in I$, then $\prod_{i \in I} L_{i}$ has an infinite disjointed subset and could therefore not be projective.

\section{REFERENCES}

1. G. Birkhoff, Lattice theory, Amer. Math. Soc. 25 (1964).

2. P. R. Halmos, Injective and projective Boolean algebras, Lattice Theory, Proc. of Symoposia in Pure Mathematics, II, (1961), 114-122.

3. Lectures in Boolean Algebras, Van Nostrand, 1963.

4. A. Nerode, Some stone spaces and recursion theory, Duke Math. J. 26 (1969), 397406.

5. N. A. Shanin, O Proizvedenii Topologicheskix Prostranstv, Trudy Matematicheskogo Instuta Imeni V. A. Steklova 24, Akad. Mauk SSR, 1948.

6. R. Sikorski, Boolean Algebras, Academic Press, 1964.

7. G. Szasz, Introduction to Lattice Theory, Academic Press, 1963.

Received August 22, 1966. The results of this paper form a part of the authors doctoral dissertation. The research was supported by the National Science Foundation Grants Nos. 4038 and 5600.

UNiversity of CALIFornia, Los ANgeles

UNIVERSITY OF MISSOURI, ST. LOUIS 


\section{PACIFIC JOURNAL OF MATHEMATICS}

\section{EDITORS}

\section{H. SAMELSON}

Stanford University

Stanford, California

\author{
J. P. JANS \\ University of Washington \\ Seattle, Washington 98105
}

\section{J. DuGUNDJI}

University of Southern California

Los Angeles, California 90007

RichaRd ARENS

University of California

Los Angeles, California 90024

\section{ASSOCIATE EDITORS}

E. F. BECKENBACH
B. H. NeUManN

F. WOLF

K. YOSIDA

\section{SUPPORTING INSTITUTIONS}

UNIVERSITY OF BRITISH COLUMBIA

CALIFORNIA INSTITUTE OF TECHNOLOGY

UNIVERSITY OF CALIFORNIA

MONTANA STATE UNIVERSITY

UNIVERSITY OF NEVADA

NEW MEXICO STATE UNIVERSITY

OREGON STATE UNIVERSITY

UNIVERSITY OF OREGON

OSAKA UNIVERSITY

UNIVERSITY OF SOUTHERN CALIFORNIA
STANFORD UNIVERSITY

UNIVERSITY OF TOKYO

UNIVERSITY OF UTAH

WASHINGTON STATE UNIVERSITY

UNIVERSITY OF WASHINGTON

AMERICAN MATHEMATICAL SOCIETY

CHEVRON RESEARCH CORPORATION

TRW SYSTEMS

NAVAL ORDNANCE TEST STATION

Mathematical papers intended for publication in the Pacific Journal of Mathematics should be typewritten (double spaced). The first paragraph or two must be capable of being used separately as a synopsis of the entire paper. It should not contain references to the bibliography. Manuscripts may be sent to any one of the four editors. All other communications to the editors should be addressed to the managing editor, Richard Arens at the University of California, Los Angeles, California 90024.

50 reprints per author of each article are furnished free of charge; additional copies may be obtained at cost in multiples of 50 .

The Pacific Journal of Mathematics is published monthly. Effective with Volume 16 the price per volume ( 3 numbers) is $\$ 8.00$; single issues, $\$ 3.00$. Special price for current issues to individual faculty members of supporting institutions and to individual members of the American Mathematical Society: $\$ 4.00$ per volume; single issues $\$ 1.50$. Back numbers are available.

Subscriptions, orders for back numbers, and changes of address should be sent to Pacific Journal of Mathematics, 103 Highland Boulevard, Berkeley 8, California.

Printed at Kokusai Bunken Insatsusha (International Academic Printing Co., Ltd.), No. 6, 2-chome, Fujimi-cho, Chiyoda-ku, Tokyo, Japan.

PUBLISHED BY PACIFIC JOURNAL OF MATHEMATICS, A NON-PROFIT CORPORATION

The Supporting Institutions listed above contribute to the cost of publication of this Journal, but they are not owners or publishers and have no responsibility for its content or policies. 


\section{Pacific Journal of Mathematics}

\section{Vol. 21, No. $3 \quad$ BadMonth, 1967}

Richard Allen Askey, A transplantation theorem for Jacobi coefficients . . . 393

Raymond Balbes, Projective and injective distributive lattices .......... 405

Raymond Balbes and Alfred Horn, Order sums of distributive lattices . . . . 421

Donald Charles Benson, Nonconstant locally recurrent functions ........ 437

Allen Richard Bernstein, Invariant subspaces of polynomially compact operators on Banach space ............................... 445

Robert F. Brown, Fixed points and fibre ................... 465

David Geoffrey Cantor, On the Stone-Weierstrass approximation theorem for valued fields ................................ 473

James Walton England, Stability in topological dynamics .............. 479

Alessandro Figà-Talamanca and Daniel Rider, A theorem on random

Fourier series on noncommutative groups.................. 487

Sav Roman Harasymiv, A note of dilations in $L^{p} \ldots \ldots \ldots \ldots \ldots \ldots . \ldots 493$

J. G. Kalbfleisch, A uniqueness theorem for edge-chromatic graphs ....... 503

Richard Paul Kelisky and Theodore Joseph Rivlin, Iterates of Bernstein polynomials .................................... 511

D. G. Larman, On the union of two starshaped sets ............... 521

Henry B. Mann, Josephine Mitchell and Lowell Schoenfeld, Properties of differential forms in $n$ real variables ...................... 525

John W. Moon and Leo Moser, Generating oriented graphs by means of team comparisons .

Veikko Nevanlinna, A refinement of Selberg's asymptotic equation ...

Ulrich Oberst, Relative satellites and derived functors of functors with additive domain ..............................

John Vincent Ryff, On Muirhead's theorem...............

Carroll O. Wilde and Klaus G. Witz, Invariant means and the Stone-Čech compactification 